\title{
A Novel Approach for Centralized 3D Radio Resource Allocation and Scheduling in Dense HetNets for 5G Control-/User-plane Separation Architectures
}

\author{
Rony Kumer Saha ${ }^{1, a, *}$, Yan Zhao ${ }^{2, b}$, and Chaodit Aswakul ${ }^{1, \mathrm{c}}$ \\ 1 Wireless Network and Future Internet Research Unit, Department of Electrical Engineering, Faculty of \\ Engineering, Chulalongkorn University, Bangkok 10330, Thailand \\ 2 International School of Engineering, Faculty of Engineering, Chulalongkorn University, Bangkok 10330, \\ Thailand \\ E-mail: arony107976@gmail.com (Corresponding author),byan.z@chula.ac.th, cchaodit.a@chula.ac.th
}

\begin{abstract}
This paper presents a centralized 3-dimensional radio resources (namely, time, frequency, and power) allocation and scheduling approach for control-plane and user-plane (C-/U-plane) separation architectures for fifth generation mobile networks. A central station is considered where schedulers of all base stations (BSs) are located. We consider a multi-tier network that comprises of a macrocell BS (MCBS), several outdoor picocell BSs, and a number of indoor femtocell BSs (FCBSs) deployed in a number of multi-storage buildings. The system bandwidth is reused in FCBSs within each building orthogonally. In contrast to the conventional almost blank subframe, we consider a fully blank subframe based time-domain enhanced intercell interference coordination to split completely C-/U-plane traffic such that the controlplane can be served only by the MCBS and the user-plane of user equipments by their respective BSs. We propose two power management schemes for FCBSs based on whether or not the coordinated multi-point communication with joint transmission (JT CoMP) is employed during off-state of a FCBS and develop a power control mechanism for both a single user and multi-user per FCBS scenarios. An optimal value of average activation factor (OAF) for a FCBS is derived to trade-off its serving capacity and transmit power saving factor. It is shown that in order to improve the network capacity, a FCBS needs to operate at an average activation factor (AAF) greater than its OAF using JT CoMP to serve neighboring on-state FCBSs during its normal off-state, whereas at an AAF less than the OAF to improve the energy efficiency. With a system level simulation, we show that the capacity of a FCBS increases, whereas its power saving factor decreases linearly with an increase in its AAF because of serving increased traffic, and an OAF of 0.5 for the capacity scaling factor $\kappa=1 / 2$ and greater than 0.5 for $\kappa<1$ are found.
\end{abstract}

Keywords:3D, 5G, centralized, control-/user-plane separation, HetNet, resource scheduling, small cell.

ENGINEERING JOURNAL Volume 21 Issue 4

Received 1 March 2017

Accepted 15 May 2017

Published 31 July 2017

Online at http://www.engj.org/

DOI:10.4186/ej.2017.21.4.287 


\section{Introduction}

\subsection{Background}

Radio resource allocation and scheduling (RRAS) plays a crucial role on achievable capacity, spectral efficiency, and energy efficiency of cellular networks. For providing a high data rate service demand and network capacity, supporting a large traffic volume, and achieving a high spectral and energy efficiencies of fifth generation $(5 \mathrm{G})$ cellular networks, the development of an effective RRAS strategy for the major 3dimensional (3D) radio resources (i.e., time, frequency, and transmit power) has been found inevitable due to their limited availability. Though most existing research addressed either 1-dimensional or 2-dimensional radio resources of these three in decentralized network architectures (i.e., distributed heterogeneous base station (BS) cellular network architectures), a number of research addressed 3D RRAS by now.

For example, authors in [1], proposed a joint power-frequency-time resource allocation algorithm for wireless mesh networks. Whereas, authors in [2] proposed a joint time-frequency-power resource allocation for low-medium-altitude platforms based Worldwide Interoperability for Microwave Access (WiMAX) networks to improve the performances and services of emergency communications. In [3], authors also proposed a joint subchannel and power allocation algorithm for the downlink of an orthogonal frequencydivision multiple access (OFDMA) femtocell (FC) and macrocell (MC) based network. However, decentralized architectures lead to scaling small cell base stations (SCBSs) with the number of user equipments (UEs) and the amount of traffic volume per unit area, which cause such architectures to suffer from a number of pitfalls, e.g. increase in network operational expense, severe inter-cell interference, and low system hardware resource utilization rate. Hence, decentralized architectures are not widely considered suitable for 5G, and the idea of centralized cellular architecture has come into being [4].

\subsection{Related Work}

Numerous proposals on centralized wireless networks are existing in literature, e.g. cloud radio access network architecture [5], wireless network cloud [6], and LightRadio [7], which are based on decoupling radio frequency and baseband processing tasks from physical nodes and shifting them to a centralized location. Similar to these architectures, authors in [4] also proposed a super BS based centralized architecture for $5 \mathrm{G}$ where the global centralized resource management center allocates resources to virtual BSs. Recently, software defined networking (SDN) has been considered as an effective centralized resource management for 5G. Authors in [8] proposed SDN-based resource management algorithms for 5G heterogeneous networks (HetNets) that exploit an SDN controller's global view of the network and take optimized resource allocation decisions. Further in [9], authors proposed to combine network function virtualization (NFV) and SDN to achieve better radio resource management performance in fourth generation $(4 \mathrm{G})$ and $5 \mathrm{G}$ HetNets.

Besides, because of an expected ultra-densification of small cells (SCs) in 5G networks, extensive research on SC energy efficiency has been ongoing. Authors in [10] proposed an energy efficient small cell activation mechanism to offload traffic from the MC to SCs in energy saving mode to reduce the total energy consumption of the network. In [11], authors quantified the tradeoff between energy consumption and throughput in a heterogeneous cellular network by considering SCBSs with four distinct power-saving modes. Authors in [12] studied the on and off (on/off) operation of SCBSs to enhance energy efficiency by applying belief propagation optimization framework into on/off operation of access points.

Further, in contrast to traditional coupled control-plane and user plane (C-/U-plane) network, C-/Uplane separation architectures (CUSAs), also termed as device centric networks [13], have been considered as a potential solution for 5G, and an extensive research is ongoing on CUSA [14-16] either by considering routing control-plane (C-plane) traffic of a SC with the SCBS itself [14] or the macrocell BS (MCBS) [1516]. Though the CUSA has been considered as one of the enabling technologies to address prospective features of 5G (e.g., a high indoor data rate, resource utilization, and energy efficiency), to our best knowledge, no existing works addressed the issue of a centralized allocation and scheduling of 3D radio resources, namely time, frequency, and power, in CUSA based 5G, which we aim to address in this paper. 


\subsection{Consideration and Contribution}

We consider a multi-tier network that comprises of a MCBS and a number of outdoor picocell BSs (PCBSs) and indoor femtocell BSs (FCBSs). All FCBSs are deployed within multi-storage buildings. A central station is considered where schedulers of all BSs are located, and the RRAS of C-plane and user-plane (U-plane) traffic of all BSs is performed. Assume that the distance between neighboring buildings and external wall penetration loss of any buildings are significant enough to overcome co-channel interference between FCBSs of neighboring buildings when reusing the same frequency in them. Hence, we consider reusing fully the whole system bandwidth in FCBSs within each building by allocating them orthogonally in any transmission time interval (TTI).

To split C-/U-plane completely such that the C-plane of all UEs is served only by the MCBS, and the U-plane of a UE is served by its respective BS, we consider a fully blank subframe (FBS) as opposed to an almost blank subframe (ABS) based time-domain enhanced inter-cell interference coordination (eICIC) to avoid co-channel cross-tier interference. We propose two transmit power management schemes for FCBSs based on whether or not coordinated multi-point communication with joint transmission (JT CoMP) is employed during the off-state of a FCBS. The JT CoMP is considered during the off-state of any FCBSs to increase the received signal strength at a UE of any FCBSs with an active traffic request to improve the network capacity. However, to improve the energy efficiency, FCBSs with no active traffic requests are considered switching off their transmit powers during their off-states. A transmit power control mechanism of FCBSs is proposed where the on-state and off-state of a FCBS is modelled as conventional on/off traffic source model for both single user and multi-user per FCBS. An optimal value of average activation factor (AAF) of a FCBS is derived to trade-off its capacity and transmit power saving.

With a system level simulation, the impact of varying the AAF of a FCBS on its capacity and transmit power saving performances is analyzed, and an optimal value of AAF (OAF) is presented. Also, to improve the serving capacity only, a FCBS needs to operate at an AAF greater than its OAF using JT CoMP, whereas an AAF less than the OAF is required to improve only the energy efficiency. In addition, the impact of varying the number of FBSs per FBS pattern period (FPP) on the overall system capacity as well as FC capacity is also shown. Since the delay from switching on/off operation of transmit power of a FCBS as well as the delay from processing UE traffic requests by exchanging control signaling between the MCBS and a FCBS influence greatly the capacity and transmit power saving of a FCBS, these effects are captured by varying AAF from 0 to 1 (i.e., switching the transmit power of a FCBS from always on-state to always off-state) over an FPP.

The paper is organized as follows. In section 2, the system architecture, operation, and mechanism of the proposed centralized radio resource allocation and scheduling (CrrAS) are presented. In section 3, we present two transmit power management schemes for FCBSs based on whether or not the JT CoMP is considered during the off-state of any FCBSs. The modelling of transmit power of any FCBSs and the effect of FBS and switching and processing delay are given in section 4. Section 5 covers the problem formation, including multi-tier network model, capacity estimation, transmit power saving factor of FCBSs, optimal average activation factor of FCBSs, effect of power management schemes on OAF, and proportional fair scheduling. Simulation parameters and assumptions are given, and performance evaluations are carried out in section 6. Finally, we conclude the paper in section 7. The list of abbreviations is given in Table 1.

\section{Proposed Centralized Radio Resource Allocation and Scheduling System Architecture, Operation, and Mechanism}

\subsection{System Architecture and Operation}

Figure 1 shows the system architecture of a multi-tier network for CrrAS where under the coverage of a MC, a number of picocells (PCs) and FCs are deployed. All FCs are considered within multi-floor buildings. For CrrAS, the scheduling of all C-plane and U-plane resources for all UEs is performed at the central station. CrrAS can help improve and optimize resource utilization globally through sharing resources between BSs, synchronization of C-plane and U-plane traffic, switching FCBS transmit power on/off, and small cell discovery for CUSA. All U-plane traffic of all UE categories are passed through their respective BSs, i.e. U-planes of all outdoor macro UEs (MUs) and indoor MUs by the MCBS, offloaded MUs by 
PCBSs, and femto UEs (FUs) by FCBSs. All these U-plane traffic is then routed through the central station, then backhauls, mobile core networks, and finally to external networks. However, C-plane of all UEs under the coverage of the MCBS is served by only the MCBS, and routed through the central station, then mobile core networks via backhauls, and finally to external networks.

Table 1. A list of abbreviations.

\begin{tabular}{ll}
\hline Abbreviation & Full Form \\
\hline 2D & 2-Dimensional \\
3D & 3-Dimensional \\
3GPP & Third Generation Partnership Project \\
4G & Fourth Generation \\
5G & Fifth Generation \\
AAF & Average Activation Factor \\
ABS & Almost Blank Subframe \\
BS & Base Station \\
C-/U-plane & Control-plane and User-plane \\
CoMP & Coordinated Multipoint \\
C-plane & Control-plane \\
CSG & Closed Subscriber Group \\
CUSA & Control-plane and User-plane Separation Architecture \\
dB & Decibel \\
dBi & Decibel Relative to an Isotropic Radiator \\
dBm & Decibel-Milliwatts \\
eICIC & Enhanced Inter-Cell Interference Coordination \\
ETSI & European Telecommunications Standards Institute \\
FBS & Fully Blank Subframe \\
FC & Femtocell \\
FCBS & Femtocell Base Station \\
FPP & FBS Pattern Period \\
FU & Femto User Equipment \\
HetNets & Heterogeneous Networks \\
JT & Joint Transmission \\
MC & Macrocell \\
MCBS & Macrocell Base Station \\
MU & Macro User Equipment \\
OAF & Optimal Average Activation Factor \\
on/off & on and off \\
PC & Picocell \\
PCBS & Picocell Base Station \\
RB & Resource Block \\
SC & Small Cell \\
SCBS & Small Cell Base Station \\
TTI & Transmission Time Interval \\
UE & User Equipment \\
U-plane & User-plane \\
\hline & \\
\hline &
\end{tabular}

For simplicity, we assume that separate ideal backhauls of negligible latency exist between any BSs and the central station for proper synchronization between C-plane and U-plane traffic as well as for helping report UE specific signals such as channel state information (CSI) or link adaptation either via the MCBS or via any SCBSs to the central station to schedule a UE to any resource blocks (RBs). The central station can be located either at the MCBS or anywhere within the MC coverage based on, e.g. the upper bound of capacity of available backhauls. An optimal number of RBs per TTI is updated and allocated by respective schedulers of the MCBS and SCBSs based on certain constraints such as control signaling traffic, long/short-term traffic demand of all MUs and FUs, average data rate demand per UE category, traffic 
characteristics, and fairness factor. To serve U-plane traffic, we consider one proportional fair scheduler for all outdoor and indoor MUs, one for all offloaded MUs, and one for all FUs per building. Also, to serve Cplane traffic, one proportional scheduler for all UEs is considered.

Because of allocating resources to all MUs and FUs at the central station, Unlike traditional decentralized CUSA [15-16], the main advantage of CrrAS is that there is no need for exchanging control signaling between C-plane and U-plane BSs for synchronization, FCBSs' transmit power switching on/off, and SC discovery and wake up mechanism required in CUSA. This results in reducing the control signaling overhead and increasing the user data traffic capacity in the backhaul.

\subsection{Mechanism}

We consider a FBS based eICIC to avoid transmitting any control signals, in contrast to a traditional ABS, to switch off the transmit power of a FCBS completely during an FBS. A static allocation of the number of FBSs per FPP, comprising 8 TTIs, by a time-domain scheduler at the central station is considered. Also a number of on/off transmit power management schemes are considered, which is detailed in a following section. All indoor MUs, offloaded MUs, and outdoor MUs in the MC coverage are allocated to RBs orthogonally. The whole system bandwidth of the MCBS is reused in FCBSs per building. Like all categories of MUs, RB allocations to all FUs within a building are considered orthogonal. If a MU is detected within any buildings, the time-domain FBS based eICIC is applied to FCBSs of that building to avoid co-channel interference between indoor MUs and FUs.

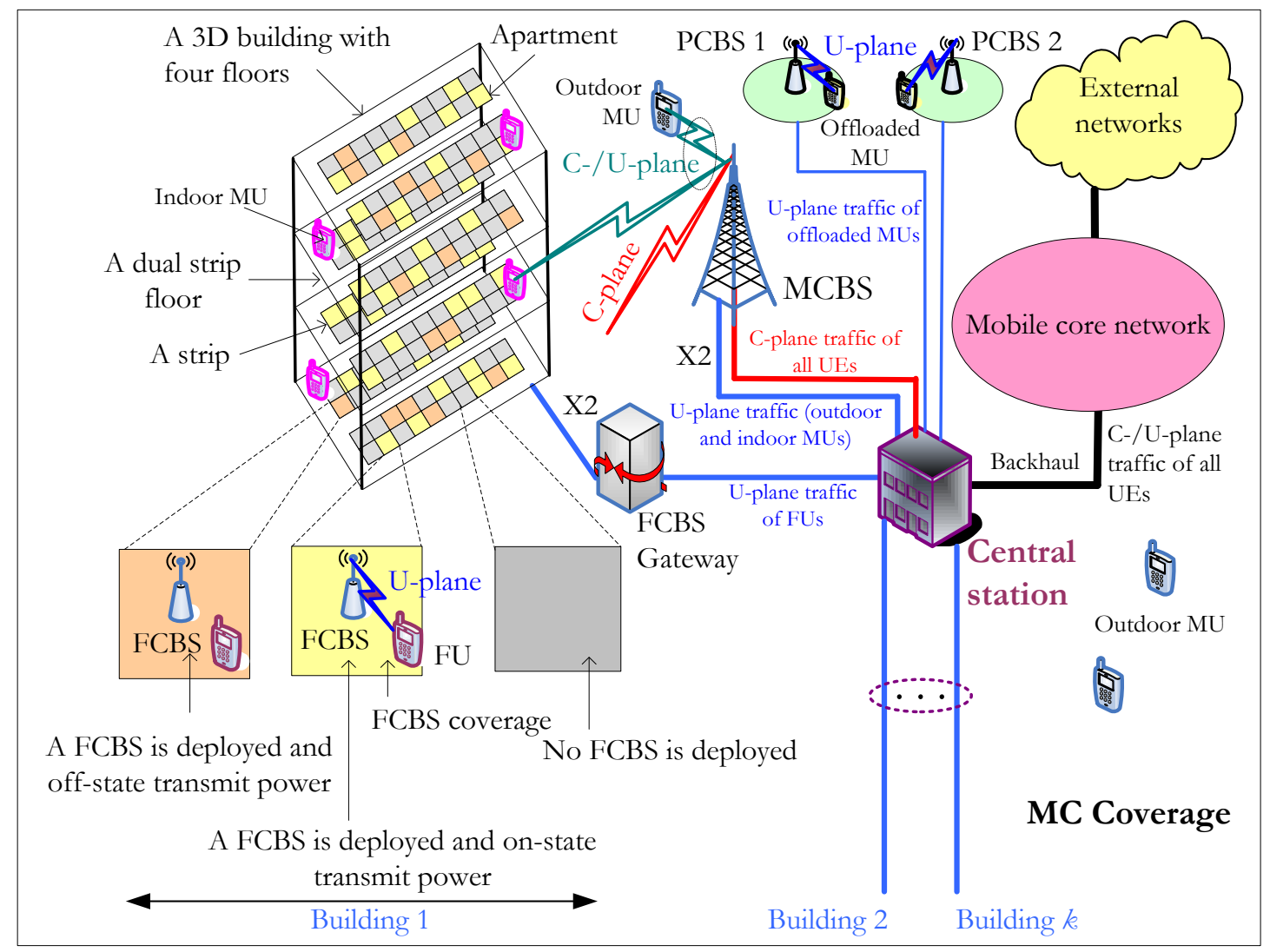

Fig. 1. System architecture of a multi-tier heterogeneous network for CrrAS with $k$ multi-storage buildings.

Note that the existence of a MU whether or not within any buildings can be determined by measuring the downlink path loss of the MU, which causes a sudden fall in received signal strength at the MU because of the presence of a high external wall penetration loss of the building when a MU enters a building. All outdoor MUs are allowed to transmit data during FBSs as well as non-FBSs. However, all indoor MUs are allowed to transmit only during FBSs, and all FUs are scheduled only during non-FBSs as shown in Fig. 2. Figure 3 shows an illustration of RRAS with respect to a FCBS. As can be seen, the transmit power of a 
FCBS has only two states, either zero or maximum, based on the FU traffic requests and other factors as shown in Fig. 4.

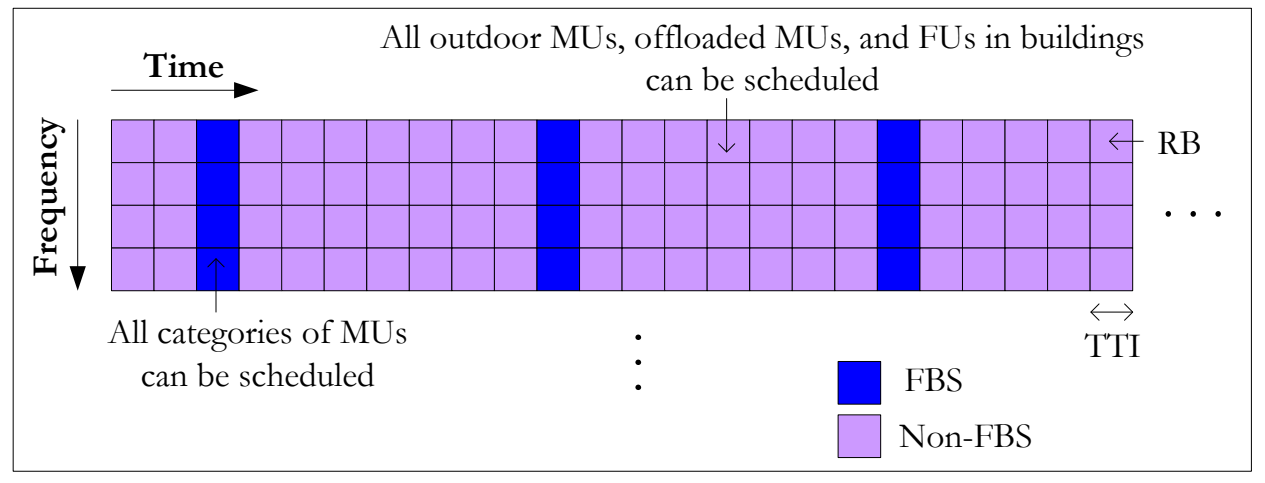

Fig. 2. FBS based time-domain eICIC.

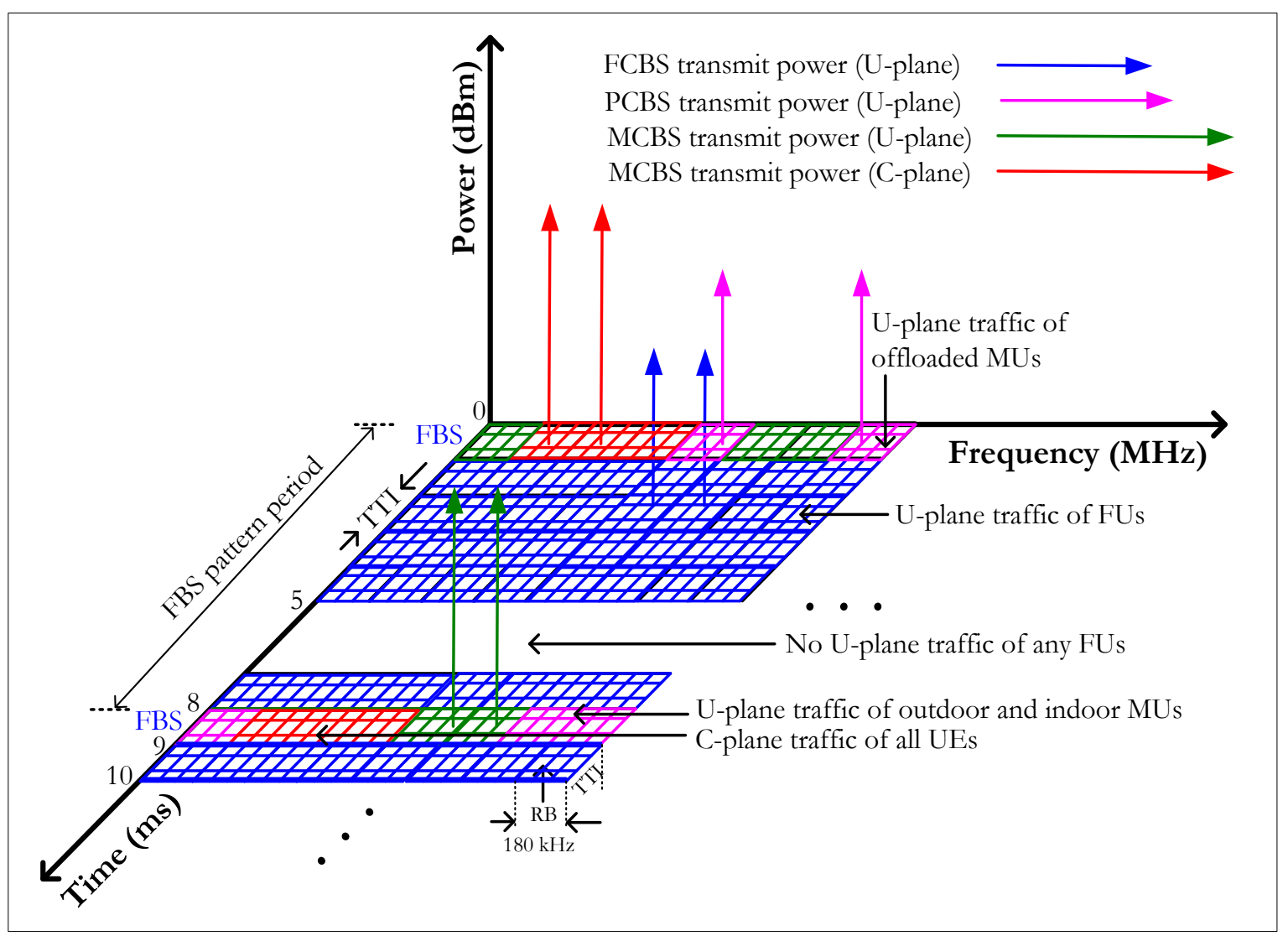

Fig. 3. An illustration of 3D RRAS concerning a FCBS.

\section{FCBS Transmit Power Management}

The transmit power of a BS has a crucial role on both spectral efficiency and energy efficiency performances. Hence, the transmit power of FCBSs needs to be managed in such a manner that can address both these aforementioned performances. A FCBS for serving U-plane traffic can monitor to detect if there is any active UE traffic requests within its coverage in every certain number of TTIs. The UE within its serving FC coverage can also be synchronized with the on/off cycle of FCBSs in order to measure the received signal strength indications (RSSIs) of all other FCBSs around its serving FCBS and to inform the frequency-domain scheduler of inactive FCBSs in order to form a suitable cooperating set of 
FCBSs to offer joint transmission (JT) coordinated multi-point (CoMP) communications to improve the UE's received signal strength.

Hence, when network capacity or spectral efficiency is concerned, cooperative or opportunistic resource allocation can be explored during the off-state of any FCBSs such that neighboring FCBSs with no active traffic requests can form a CoMP set to help increase the received signal strength of any FCBSs with an active traffic request. In contrast, when energy efficiency is concerned, FCBSs with no active traffic requests can be muted for transmission by switching their transmit power off. Hence, based on whether or not a FCBS is considered transmitting power during its off-state, we propose two power management schemes of any FCBSs as described in the following.

\subsection{Without Exploiting JT CoMP during Off-state of a FCBS Transmit Power}

In this scheme, FCBSs are not always active. Rather, each FCBS is switched on/off based on active data traffic requests. An FCBS is considered switching on as long as an active data traffic session exists, and switching it off after a certain number of TTIs as shown in Fig. 4,which can be modeled as the on/off traffic source model. Switching on/off any FCBSs is governed by the respective scheduler at the central station. When there is no further active data requests from a UE (i.e., FU) under the coverage of a FCBS, the scheduler then sends a power-off message to that FCBS to keep its transmit power switched off until any further request is made by any UEs. In switching on an FCBS, a UE first sends a random access channel request to the MCBS in the uplink. Assuming that a mechanism exists for selecting an appropriate FCBS by the MCBS, the MCBS informs of the UE request to the corresponding frequency-domain scheduler at the central station. The scheduler then sends a power-on message to the FCBS to which the FU may get connected with in the downlink and creates necessary data bearers through that FCBS. The scheduler also informs the UE over the backhaul via the MCBS to create relevant radio resource control (RRC) connections with the FCBS. After acknowledging the RRC connection complete message from the FCBS, the UE then starts communicating via the FCBS to mobile core networks and then to external networks.

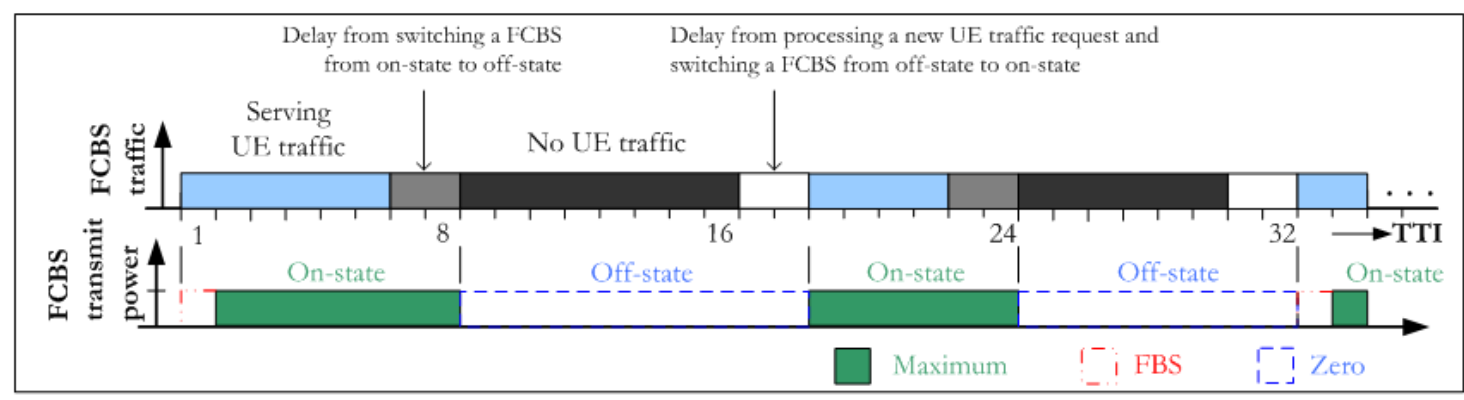

Fig. 4. An illustration of the transmit power on/off scheme of a FCBS without exploiting JT CoMP during its off-state.

\subsection{With Exploiting JT CoMP during Off-state of a FCBS Transmit Power}

According to [17], there is some unused time between transmitted packets of a traffic service, which can be used for other traffic services. Since we explore at connection levels (i.e., UE traffic requests) rather than packet levels, inactive FCBSs can serve other active FCBSs using JT CoMP during their off-states following the procedure proposed and described in the following. When an on-state FCBS does not have any data to serve to its own serving UE, it can switch from its on-state to off-state to save power so that energy efficiency can be improved. However, if the demand is to enhance spectral efficiency or network capacity rather than energy efficiency, an off-state FCBS can serve other neighbor UEs to increase their throughputs as shown in Fig. 5. Neighboring FCBSs with no active traffic requests can form a CoMP set for JT to a UE of the FCBS with active traffic requests. All FCBSs in a CoMP set transmit the same data to the UE such that the signal strength, and hence the capacity can be improved from multi-point transmissions. An illustrative mechanism for JT CoMP is discussed in the following. 


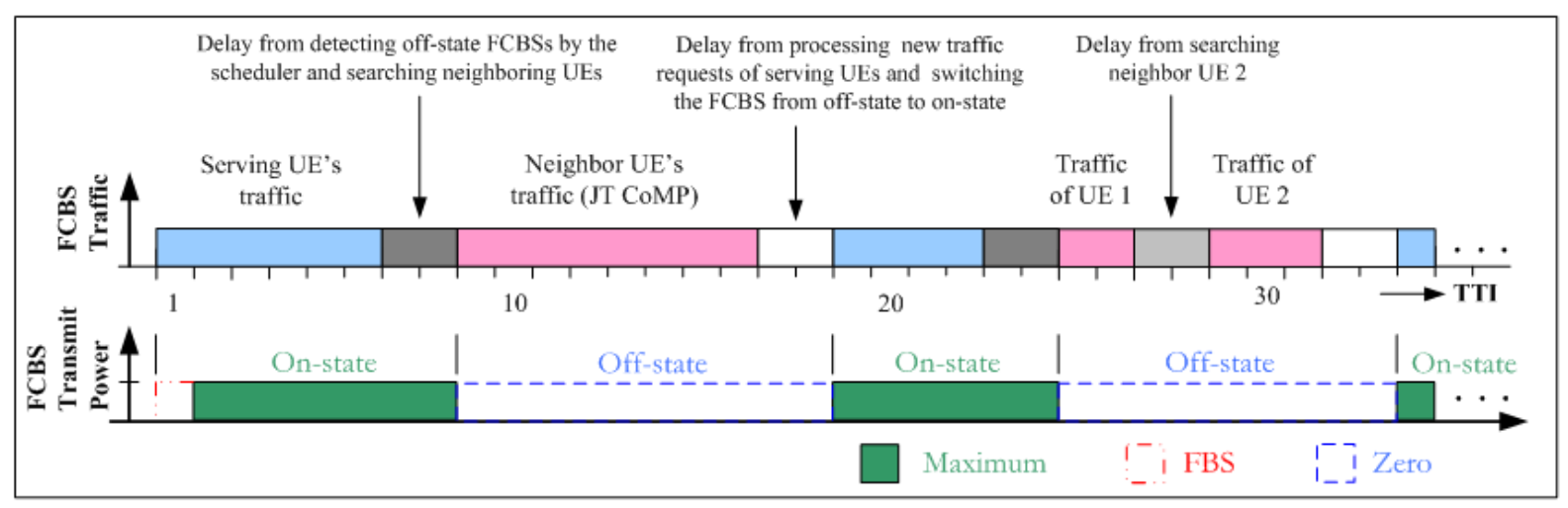

Fig. 5. An illustration of the transmit power on/off scheme of a FCBS with exploiting JT CoMP during its off-state.

An off-state FCBS serves a UE of one of its nearest neighbor FCBSs either at the same frequency as that of the serving FCBS or at a different frequency in any TTIs based on the operator's policy for an efficient bandwidth utilization. Typically, the frequency-domain scheduler has the knowledge of physical cell identity (PCI) and on/off-state status of each FCBS in order to determine and inform any off-state FCBSs to serve during its off-state. Since the scheduler has all the UEs' CSIs and traffic data rate demands, if there exists more than one UE to serve during its off-state, the off-state FCBS is being informed by the scheduler to serve the UE with the maximum service data rate demand, for example. Further, if all the neighbor UEs has the same service data rate demand, the off-sate FCBS may serve any UEs by choosing randomly.

Once an off-state FCBS is chosen to serve any neighbor UEs, it continues to serve the same UE so long as either that UE's data transmission is finished, or until there is a new request from its own serving UE. Hence, even though other neighbor UEs' either CSIs or data rate demands are higher than that of the UE, which has been already chosen by the off-state FCBS, it continues to serve it in order to reduce control signaling overhead (Fig. 5). Also, if during the off-state, the existing UE's (UE1) traffic is finished to serve, the off-state FCBS starts searching to serve another neighboring UE (UE2) if any, and continues to serve as long as any new traffic requests from its own serving UEs is not made. When any such new request is made, the FCBS then stops serving the neighboring UE and starts serving its own UE. All these activities are shown in Fig. 5 where UE 1 and UE 2 are neighboring UEs served by a FCBS during its off-state.

So, based on switching on/off operations (i.e., if there is any FU traffic requests) of transmit power of FCBSs during the off-state, inactive FCBSs can be considered transmitting other neighboring active FUs at any RB $i$ in TTI $t$ to enhance the overall network capacity and spectral efficiency further while keeping the energy efficiency within a limited range. Hence, a tradeoff between the network capacity and energy efficiency is needed, which is addressed in a later section by deriving an OAF.

\section{Modeling FCBS Transmit Power and Effect of FBS and Switching and Processing Delay}

\subsection{FCBS Transmit Power Modeling}

The $3^{\text {rd }}$ Generation Partnership Project (3GPP) European Telecommunications standards Institute (ETSI) traffic model for non-real time Internet data has three layers, namely session, packet call, and packets [18]. BSs need to be active at the upper most layer, i.e. session layer, while a UE is communicating through them. According to [18], sessions or call arrivals can be modelled as a Poisson process. For simplicity, we assume that a BS activity is directly proportional to the cumulative traffic activity from its UEs. Hence, a U-plane BS (i.e., FCBS) transmit power on-state and off-state can be modelled as exponentially distributed continuous time Poisson process such that the amount of time any FCBSs spent on each state is exponentially distributed. Since given the present state, the future state is independent of the past state, the on-state and off-state transmit powers of any FCBSs can be modelled as two state Markov chain as shown 
in Fig. 6, where $\lambda$ denotes the power off-state to on-state transition rate, and $\mu$ denotes the power on-state to off-state transition rate of any FCBSs.

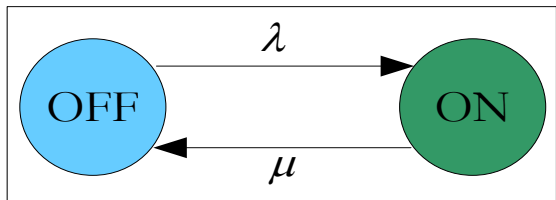

Fig. 6. Two-state on/off transmit power model for a FCBS.

The randomness in switching any FCBS's transmit power to on/off states can be modelled by the average duration of each state such that the average time at an on-state is given by $1 / \mu$ and at an off-state by $1 / \lambda$ [17]. Hence, the AAF, which is defined as the probability that a FCBS is at the on-state, is given by,

$$
\begin{aligned}
\alpha & =P(\text { on }- \text { state }) \\
=(1 / \mu) /((1 / \lambda)+(1 / \mu)) & \\
& =\lambda /(\lambda+\mu)
\end{aligned}
$$

Hence,

$$
\begin{aligned}
P(\text { off }- \text { state }) & =1-\alpha \\
& =\mu /(\lambda+\mu)
\end{aligned}
$$

For a single FU per FCBS, because a FU also has two states (i.e., there is either a traffic request or not at all), the FU state diagram is the same as its FCBS's on/off state diagram (Fig. 6). Hence, for a single FU per FCBS, the average on/off state probabilities of transmit power of any FCBSs are given by Eq. (1) and Eq. (2).

However, for multi-user per FCBS, finding the values of on-state and off-state durations is not immediate since there exists more than one UE, and the FCBS may have to be active with an activity factor of $100 \%$ at the extreme case. To find the on/off state probabilities of FCBS for such multi-user per FCBS scenario, a more general and simple way is to calculate the probability of no UE traffic requests in progress such that the probability of off-state can be given by,

$$
P(\text { off }- \text { state })=1-p(0)
$$

And the probability of on-state can be given by,

$$
P(\text { on-state })=p(1)+p(2)+\cdots+p\left(N_{s}\right)
$$

where $p(0), p(1), p(2), \cdots, p\left(N_{s}\right)$ represent the state probability for $i_{s}=0,1,2, \ldots, N_{s} . N_{s}$ is the number of users with in-progress traffic with the FCBS. The values of these probabilities can be found following the Birth-Death process as follows.

Consider that there are $M_{s}$ users per FCBS of which $N_{s}$ users have in-progress traffic with the FCBS at any time $t$ as shown in Fig. 7. Let $\lambda_{N_{s}}$ and $\mu_{N_{s}}$ denote the birth rate and the death rate respectively. Then, according to [17, 19-20], the followings hold.

$$
\begin{gathered}
\lambda_{N_{s}}= \begin{cases}\left(M_{s}-N_{s}\right) \lambda, & 0 \leq N_{s} \leq M_{s} \\
0, & \text { otherwise }\end{cases} \\
\mu_{N_{s}}=N_{s} \cdot \mu
\end{gathered}
$$


And the probabilities for any $N_{s}$ (Fig. 7) can be given by,

$$
p\left(N_{s}\right)=p(0)(\lambda / \mu)^{N_{s}}\left(\begin{array}{l}
M_{s} \\
N_{s}
\end{array}\right)
$$

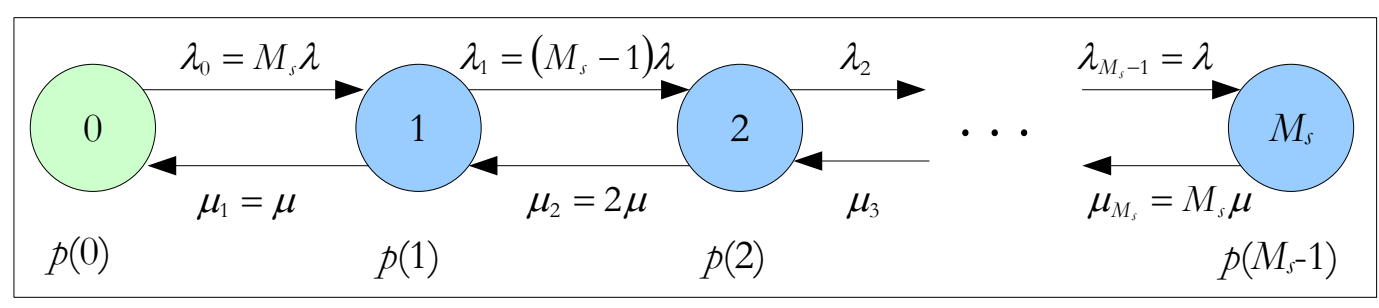

Fig. 7. Occupancy state diagram of a FCBS.

Such that,

$$
\begin{gathered}
p(0)=1 /(1+\lambda / \mu)^{M_{s}} \\
P(\text { off }- \text { state })=p(0) \\
=1 /(1+\lambda / \mu)^{M_{s}} \\
\alpha=P(\text { on }- \text { state }) \\
=1-1 /(1+\lambda / \mu)^{M_{s}}
\end{gathered}
$$

Hence, for a particular duration of time $T_{\mathrm{s}}$, the average on-state duration of any FCBSs is given by,

$$
t_{\text {on-state }}=\alpha \times T_{\mathrm{s}}
$$

Similarly, the off-state duration is given by,

$$
t_{\text {off-state }}=(1-\alpha) \times T_{\mathrm{s}}
$$

For evaluation, we consider an average value of probabilities, e.g. given by Eq. (1) and Eq. (2), per FCBS per FPP so that an average value is estimated in ratio of the number of TTIs per FPP per FCBS.

\subsection{Effect of FBS and Switching and Processing Delay}

Let $\alpha_{\mathrm{on} \rightarrow \text { off }}$ denote an average aggregate delay per FPP T from switching on-state to off-state of any FCBSs, whereas $\alpha_{\text {off } \rightarrow \text { on }}$ denote delay from switching off-state to on-state, for traffic request processing of any FUs, e.g. discovery of any FCBSs and C-/U-plane cooperation for synchronization. Let $\alpha_{\mathrm{fbs}}$ denote percentage duration of FBSs, and $\alpha_{\mathrm{s}}$ and $\alpha_{\mathrm{ns}}$ denote percentage average durations of any in-progress active and inactive FU traffic respectively per FPP $T$.

The average on-state duration of any FCBSs can be given by,

$$
\begin{aligned}
t_{\text {on-state }} & =\alpha \times T \\
& =\left(\alpha_{\mathrm{s}}+\alpha_{\mathrm{on} \rightarrow \text { off }}\right) \times T
\end{aligned}
$$

Similarly, the average off-state duration of any FCBSs is given by,

$$
\begin{aligned}
t_{\text {off-state }} & =(1-\alpha) \times T \\
& =\left(1-\left(\alpha_{\mathrm{s}}+\alpha_{\mathrm{on} \rightarrow \text { off }}\right)\right) \times T
\end{aligned}
$$


Equivalently,

$$
t_{\text {off }- \text { state }}=\left(\alpha_{\text {ns }}+\alpha_{\text {off } \rightarrow \text { on }}+\alpha_{\text {fbs }}\right) \times T
$$

Such that,

$$
T=t_{\text {on-state }}+t_{\text {off }- \text { state }}
$$

\section{Problem Formulation}

\subsection{Multi-Tier Network Model}

Consider that there are $M \mathrm{RBs}$ in the system bandwidth, and $N$ MUs in the system. Let $S_{\mathrm{P}}$ denote the number of PCs in the MC coverage. Consider that the number of offloaded MUs is uniformly distributed in the interval [1, $\left.U_{\text {OFL }}\right]$. If all PCs have an equal number of offloaded MUs $U_{\mathrm{P}}$, i.e. $\forall q U_{\mathrm{P}}^{q}=U_{\mathrm{P}}$, then the total number of offloaded MUs, $U_{\mathrm{OFL}}=S_{\mathrm{P}} \times U_{\mathrm{P}}$. However, in general, $U_{\mathrm{P}}^{g}$ is a random variable, which varies from one PC to another, and the realization of $U_{\mathrm{P}}^{a}$ for a PC is mutually independent from the others. If $\mu_{\mathrm{MI}}$ denotes the ratio of the number of indoor MUs, then the total number of indoor MUs is $U_{\mathrm{MI}}=\mu_{\mathrm{MI}} \times N$, outdoor MUs is $U_{\mathrm{MO}}=N-U_{\mathrm{OFL}}-U_{\mathrm{MI}}$, and MUs served by the $\mathrm{MC}$ is $U_{\mathrm{M}}=U_{\mathrm{MO}}+U_{\mathrm{MI}}$.

Let $\mathrm{NM}$ denote the set of indices of all MUs such that $\mathrm{NM}=\{1,2,3, \ldots, \mathrm{N}\}$. Denote $N M O, N P$, and NMI respectively the set of indices of all outdoor MUs, offloaded MUs, and indoor MUs. Note that NM is partitioned randomly into three disjoint subsets NMO, NP, and NMI. Let L denote the maximum number of buildings in a MC coverage, and SF denote the number of active FCs in each building. Assuming that SF is the same for all buildings, the total number of active FCs in the system is SFS $=\mathrm{L} \times \mathrm{SF}$. Consider that the number of FUs in buildings are independent and uniformly distributed in the interval [1, UF]. In general, UF is a random variable, which varies from one building to another, and the realization of UF for a building is mutually independent from the others where a realization is defined as a simulation run time.

Let $U_{\mathrm{F}}^{w}$ denote the number of FUs served by a FC $S_{\mathrm{F}}^{m}$ in a building such that $\forall w U_{\mathrm{F}}^{m} \in\left[0, U_{\mathrm{F}, \max }^{m}\right]$. If all FCs have an equal number of FUs $U_{\mathrm{FC}}$, i.e. ${ }^{w} U_{\mathrm{F}}^{m}=U_{\mathrm{FC}}$, then the total number of FUs in any buildings, $\mathrm{UF}=\mathrm{SF} \times \mathrm{UFC}$. However, in general, ${ }_{\mathrm{F}}^{w}$ is a random variable, which varies from one FCBS to another, and the realization of $U_{\mathrm{F}}^{\mu}$ for a FCBS is mutually independent from the others. If each FC in a building serves one $\mathrm{UE}$, i.e. $U_{\mathrm{FC}}=1$, the total number of FUs in a building is $\mathrm{UF}=\mathrm{SF}$, and in the system is $\mathrm{UFS}=\mathrm{L} \times \mathrm{UF}$. Let NF denote the set of all FU indices in a building such that $\mathrm{NF}=\{1,2,3, \ldots, \mathrm{UF}\}$.

The realization of MUs served by the MC and PCs are not mutually independent since MUs served by PCs are MUs offloaded from the MCBS, and the schedulers have a complete knowledge when a MU is offloaded. FC buildings and PCs are located randomly and uniformly in the MC area. The indoor MUs are distributed randomly and non-uniformly within buildings. All outdoor MUs, offloaded MUs, and FUs are distributed randomly and uniformly within their respective BSs' coverage area.

Let $\boldsymbol{T}$ denote simulation run time with the maximum time of $\mathcal{Q}$ (in time step each lasting $1 \mathrm{~ms}$ ) such that $\boldsymbol{T}=\{1,2,3, \ldots, Q\}$. Let $\boldsymbol{T}_{\mathrm{FBs}}$ denote a set of FBS indices over all FPPs for $Q$ TTIs, and $T_{\mathrm{FPP}}$ denote the number of subframes per FPP such that $\boldsymbol{T}_{\mathrm{FBS}} \subseteq \boldsymbol{T}$ and $\boldsymbol{T}_{\mathrm{FBS}}=\left\{t . t=\left(v \times T_{\mathrm{FPP}}\right)+n ; v=0,1,2, \ldots, Q / T_{\mathrm{FPP}}\right.$; $\left.n=1, \ldots, T_{\mathrm{FBS}}\right\}$ where $T_{\mathrm{FBS}}=1,2, \ldots, T_{\mathrm{FPP}}$ corresponds to FBS patterns $\alpha_{\mathrm{fbs}}=1 / T_{\mathrm{FPP}}, 2 / T_{\mathrm{FPP}}, \ldots, T_{\mathrm{FPP}} / T_{\mathrm{FPP}}$ respectively. Let $t_{\mathrm{FBS}}$ and $t_{\mathrm{non}-\mathrm{FBS}}$ denote respectively an FBS and a non-FBS such that $t_{\mathrm{FBS}} \in \boldsymbol{T}_{\mathrm{FBS}}$ and $t_{\text {non-FBS }} \in \boldsymbol{T} \backslash \boldsymbol{T}_{\mathrm{FBS}}$.

Let $\boldsymbol{t}_{\text {on-state }}$ denote a set of subframes over all FPPs for $\boldsymbol{T}$, and $t_{\text {on-state }}$ denote the number of subframes per FPP such that $\boldsymbol{t}_{\text {on-state }} \subseteq \boldsymbol{T}$. In general, $t_{\text {on-state }}$ is an integer random variable, which varies from one $T_{\mathrm{FPP}}$ to another for $Q$ TTIs and depends mainly on in-progress active UE traffic requests and characteristics over any $T_{\mathrm{FPP}}$.

\subsection{Capacity Estimation}


Let $d_{\mathrm{MU}}, d_{\mathrm{PC}}$, and $d_{\mathrm{FCL}}$ denote respectively the distances of any MUs, PCs, and buildings from the MCBS, and $d_{\mathrm{FC}}$ denote the distance between a FCBS and a FU. The distances of all UEs of each category in a realization are generated following the respective distribution functions as mentioned earlier. The received signal-to-interference-plus-noise ratio (SINR) for a UE at RB $i$ in TTI $t$ at power $p$ can be expressed as

$$
\rho_{t, i, p}=\left(P_{t, i, p}^{\mathrm{tr}} /\left(N_{t, i, p}^{\mathrm{s}}+I_{t, i, p}\right)\right) \times H_{t, i, p}
$$

where $P_{t, i, p}^{\mathrm{tr}}$ is the transmission power; $N_{t, i, p}^{\mathrm{s}}$ is the noise power; $I_{t, i, p}$ is the total interference signal power; and $H_{t, i, p}$ is the link loss for a link between a UE and a BS at RB $i$ in TTI $t$ at power $p$, which can be expressed in $\mathrm{dB}$ as

$$
H_{t, i, p}(\mathrm{~dB})=\left(G_{t}+G_{r}\right)-\left(L_{F}+P L_{t, i, p}\right)+\left(L S_{t, i, p}+S S_{t, i, p}\right)
$$

where $\left(G_{t}+G_{r}\right)$ and $L_{F}$ are respectively the total antenna gain and connector loss, and $L S_{t, i, p}, S S_{t, i, p}$, and $P L_{t, i, p}$ respectively denote shadowing effect, small-scale Rayleigh fading or Rician fading, and distance dependent path loss between a BS and a UE at RB $i$ in TTI $t$ at power $p$. Let $\beta$ denote implementation loss factor. Using Shannon's capacity formula, a link throughput at RB $i$ in TTI $t$ at power $p$ in bps per Hz is given by [21-22],

$$
\sigma_{t, i, p}\left(\rho_{t, i, p}\right)=\left\{\begin{array}{ll}
0, & \rho_{t, i, p}<-10 \mathrm{~dB} \\
\beta \log _{2}\left(1+10\left(\rho_{t, i, p}(\mathrm{~dB}) / 10\right)\right), & -10 \mathrm{~dB} \leq \rho_{t, i, p} \leq 22 \mathrm{~dB} \\
4.4, & \rho_{t, i, p}>22 \mathrm{~dB}
\end{array}\right\}
$$

Since any FCBSs transmits only u-plane traffic during its on-state, using Eq. (13), the aggregate average capacity of FCBSs is given by,

$$
\sigma_{\mathrm{FC}}=\sum_{t=1}^{Q} \sum_{i=1}^{M} \alpha_{\mathrm{s}} \times \sigma_{t, i, p}\left(\rho_{t, i, p}\right)
$$

where $\sigma_{t, i, p}\left(\rho_{t, i, p}\right)$ denotes the throughput response of all FUs in $t \in \boldsymbol{t}_{\text {on-state }}$ Over $M$ RBs.

The total system capacity over the whole system bandwidth for $\mathcal{Q}$ TTIs can be expressed as the sum throughput of all UEs as follows.

$$
\sigma_{\mathrm{S}}=\sum_{t=1}^{Q} \sum_{i=1}^{M} \sigma_{t, i, p}\left(\rho_{t, i, p}\right)
$$

where $\sigma$ and $\rho$ are responses of all MUs in $t \in \boldsymbol{T}_{\mathrm{FBS}}$, outdoor and offloaded MUs in $t \in \boldsymbol{T} \backslash \boldsymbol{T}_{\mathrm{FBS}}$, and all FUs in $t \in \boldsymbol{t}_{\text {on-state. }}$

\subsection{FCBS Transmit Power Saving and Optimal Average Activation Factors}

5.3.1. FCBS transmit power saving factor

Using Eq. (13) and Eq. (14), the power saving factor of any FCBSs can be given by the average on-state and off-state durations as follows.

$$
\begin{aligned}
\eta= & t_{\text {off }- \text { state }} /\left(t_{\text {on-state }}+t_{\text {off }- \text { state }}\right) \\
& =((1-\alpha) \times T) / T \\
& =1-\alpha
\end{aligned}
$$


Hence, the percentage power saving factor is given by,

$$
\% \eta=(1-\alpha) \times 100
$$

5.3.2. FCBS optimal average activation factor

Since both the capacity and power saving factor of any FCBSs are proportional to AAF, an OAF that can trade-off these demands can be defined as its corresponding value of a point where the capacity and power saving responses are equal so that the optimization problem can be formulated as follows.

$$
\begin{array}{ll}
\operatorname{maximize} & \alpha \\
\text { subjectto } & \eta-\left(\sigma_{\mathrm{FC}} / \sigma_{\mathrm{FC}, \max }\right)=0
\end{array}
$$

where $\sigma_{\mathrm{FC} \text {,max }}$ denotes the maximum capacity of FUs in $t \in \boldsymbol{T}$ over $M \mathrm{RBs}$, whereas $\sigma_{\mathrm{FC}}$ implies the actual aggregate capacity of FCs as given by Eq. (20).

From Eq. (20), since $\sigma_{\mathrm{FC}}$ is a function of link quality irrespective of the value of $\alpha_{\mathrm{s}}, \sigma_{\mathrm{FC} \text {,max }}$ can be defined and fixed in prior as a system parameter by setting the maximum value of the average link quality of an FU $\sigma_{\mathrm{lq}}$ based on, e.g. the operating band of FCBSs such that $\sigma_{\mathrm{FC}} \leq \sigma_{\mathrm{FC} \text {,max }}$. The inequality comes from the fact that the average actual link quality of any FUs is upper bounded by $\sigma_{\mathrm{lq}}$, which is incorporated in $\sigma_{\mathrm{FC}}$ by a capacity scaling factor $\kappa$ such that $0<\kappa \leq 1$.

The solution of the above optimization problem for $\alpha \cong \alpha_{\mathrm{s}}$ can be found as follows, which is proved in the following.

$$
\alpha^{*}=1-\left(\sigma_{\mathrm{FC}} / \sigma_{\mathrm{FC}, \max }\right)
$$

Proof. From Eq. (20),

$$
\sigma_{\mathrm{FC}, \max }=(Q \times M) \times \alpha_{\mathrm{s}} \times \sigma_{\mathrm{lq}}
$$

Applying the constraint,

$$
\begin{gathered}
\eta=\sigma_{\mathrm{FC}} / \sigma_{\mathrm{FC}, \max } \\
\Rightarrow 1-\alpha=\sigma_{F C} / \sigma_{F C \text {,max }} \\
\Rightarrow \alpha=1-\left(\sigma_{\mathrm{FC}} / \sigma_{\mathrm{FC}, \max }\right)
\end{gathered}
$$

From Eq. (13),

$$
\alpha=\alpha_{\mathrm{s}}+\alpha_{\mathrm{on} \rightarrow \mathrm{off}}
$$

Assuming $\alpha_{\mathrm{s}} \gg \alpha_{\mathrm{on} \rightarrow \text { off }}$ such that $\alpha \cong \alpha_{\mathrm{s}}$. Hence, by omitting the inequality with $\kappa$ as explained above and using Eq. (20), $\sigma_{\mathrm{FC}}$ can be expressed in terms of $\sigma_{\mathrm{FC}, \max }$ as follows.

$$
\sigma_{\mathrm{FC}}=\kappa \times \sigma_{\mathrm{FC} \text {,max }}
$$

Further from Eq. (25), the solution for $\alpha=\alpha^{*}$ can be found as follows.

$$
\begin{gathered}
\alpha^{*} \cong 1-\left(\left(\kappa \times \sigma_{\mathrm{FC} \text { max }}\right) / \sigma_{\mathrm{FC} \text {,max }}\right) \\
\Rightarrow \alpha^{*} \cong 1-\kappa
\end{gathered}
$$

For $\kappa=1 / 2$, the above solution becomes as follows. 


$$
\alpha^{*} \cong 1 / 2
$$

\subsubsection{Effect of power management schemes on OAF}

When considering JT CoMP to improve network capacity further from that can be achieved at $\alpha=\alpha^{*}$, the solution of the above optimization problem can be given by,

$$
\begin{gathered}
\alpha_{\mathrm{nc}}^{*}>\alpha^{*} \\
\Rightarrow \alpha_{\mathrm{nc}}^{*}>1-\left(\sigma_{\mathrm{FC}} / \sigma_{\mathrm{FC}, \max }\right)
\end{gathered}
$$

where $\alpha_{\mathrm{nc}}^{*}$ denotes an OAF that corresponds to the improved network capacity set by the network operator.

Similarly, when considering energy efficiency to improve further from that can be achieved at $\alpha=\alpha^{*}$, the solution for an OAF can be given by,

$$
\alpha_{\mathrm{ec}}^{*}<1-\left(\sigma_{\mathrm{FC}} / \sigma_{\mathrm{FC}, \max }\right)
$$

where $\alpha_{\mathrm{ec}}^{*}$ denotes an OAF that corresponds to the improved energy efficiency also set by the network operator. Note that a FCBS operates as a typical single network entity to serve UEs only within its coverage when considering its AAF $\alpha$ less than that of its OAF $\alpha^{*}$. Hence, there is no need for JT CoMP to address energy efficiency improvement.

\subsection{Proportional Fair Scheduling}

Since proportional fair scheduler provides an optimal trade-off between fairness and throughput performances, we consider it to schedule time and frequency resources among UEs. Based on the current and past average throughputs of a UE, it schedules a UE $x_{i}(t)$ in TTI $t$ at RB $i$ with the maximum performance metric given by [23],

$$
x_{i}(t)=\underset{x}{\arg \max }\left(\sigma_{x, i}(t) / \widetilde{\sigma}_{x, i}(t)\right)
$$

where $\sigma_{x, i}(t)$ and $\tilde{\sigma}_{x, i}(t)$ represent respectively the current and past average throughputs of UE $x$ at RB $i$ in T'TI $t$. The past average throughput $\widetilde{\sigma}_{x, i}(t)$ at RB $i$ is updated in every TTI as follows [24] where $t_{\mathrm{c}}$ denotes adjustable time constant.

$$
\tilde{\sigma}_{x, i}(t+1)=\left\{\begin{array}{ll}
\widetilde{\sigma}_{x, i}(t)\left(1-1 / t_{\mathrm{c}}\right)+1 / t_{\mathrm{c}} \sigma_{x, i}(t), & x=x_{i}(t) \\
\widetilde{\sigma}_{x, i}(t)\left(1-1 / t_{\mathrm{c}}\right), & x \neq x_{i}(t)
\end{array}\right\}
$$

\section{Simulation Parameters, Assumptions, Performance Results}

\subsection{Simulation Parameters and Assumptions}

The default simulation parameters and assumptions used for the system level simulation are listed in Table 2. Unless stated explicitly, the default value for any parameters is used from Table 2. Note that rather than choosing sophisticated but computationally complex theoretical models such as ray tracing, we consider empirical simplified path loss model for indoor FCs and assume the similar mechanisms for the dual-strip model for evaluating the performance of FCs [25] recommended by the 3GPP. We consider that FCs in all buildings experience the similar signal propagation characteristics. Hence, there would not be any significant deviation in the performance results from using empirical models that do not necessarily guarantee the transportability between environments. Also, eICIC is considered to address mainly the cross-tier interference between indoor MUs and femto-tier. 


\subsection{Performance Results}

For simplicity in evaluation, because of considering the average value of probability of each factor over an FPP, influencing on-state and off-state durations, we consider one FCBS for performance evaluation. However, this will not affect the performance trends as indoor channels are less susceptible to Doppler Effect and delay spread because of less movement of objects and small coverage of a $\mathrm{FC}$. We define $\sigma_{\mathrm{FC}, \max }$ as the average sum throughputs of FUs in $t \in \boldsymbol{T}$ over $M$ RBs per realization. Since we consider one realization per $\mathrm{FBS}, \sigma_{\mathrm{FC}, \max }=\sigma_{\mathrm{FC}}$.

From Fig. 8, it can be found that the capacity of a FC increases linearly with an increase in its AAF $\left(\alpha \cong \alpha_{\mathrm{s}}\right.$ ), which influences directly the overall system level capacity. The maximum capacity of a FC attains when the FC serves over the whole FPP. However, this is possible only if there is no existence of MU within the building such that the FCBS can be allowed to transmit in all TTIs of any FPPs. In Fig. 8, the overall system capacity of all UEs is shown for FBS $=1$. However, irrespective of the number of FBSs per

Table 2. Default simulation parameters and assumptions.

\begin{tabular}{|c|c|c|c|}
\hline \multicolumn{3}{|c|}{ Parameter and Assumptions } & Value \\
\hline \multicolumn{3}{|c|}{ E-UTRA simulation case ${ }^{1}$} & 3GPP case 3 \\
\hline \multicolumn{3}{|c|}{ Cellular layout $^{2}$ and Inter-site distance 1,2} & $\begin{array}{l}\text { Hexagonal grid, dense urban, } 3 \text { sectors per MC } \\
\text { site and } 1732 \mathrm{~m}\end{array}$ \\
\hline \multicolumn{3}{|c|}{ Carrier frequency ${ }^{2}$ and transmit direction } & $2 \mathrm{GHz}$ and downlink \\
\hline \multicolumn{3}{|c|}{ System bandwidth and cells } & $5 \mathrm{MHz}$ and $1 \mathrm{MC}, 2 \mathrm{PCs}$, and $1 \mathrm{FC}$ \\
\hline \multicolumn{3}{|l|}{ Type of FCs } & Closed subscriber group (CSG) \\
\hline \multicolumn{3}{|c|}{ Total BS transmit power ${ }^{1}(\mathrm{dBm})$} & 46 for $\mathrm{MC}^{1}, 37$ for $\mathrm{PC}^{1}, 20$ for $\mathrm{FC}^{1}$ \\
\hline \multicolumn{4}{|c|}{ Frequency selective Rayleigh (MC and PC), Rician (FC) } \\
\hline \multirow[t]{4}{*}{ Path loss (PL) } & \multirow[t]{2}{*}{ MCBS and a UE 1} & outdoor MU & $P L(\mathrm{~dB})=15.3+37.6 \log _{10} R, R$ is in $\mathrm{m}$ \\
\hline & & indoor MU & $P L(d B)=15.3+37.6 \log _{10} R+L_{\text {ow }}$ \\
\hline & PCBS and a UE 1 & & $P L(\mathrm{~dB})=140.7+36.7 \log _{10} \mathrm{R}, \mathrm{R}$ is in $\mathrm{km}$ \\
\hline & FCBS and a UE 1,2 & & $P L(\mathrm{~dB})=127+30 \log _{10}(\mathrm{R} / 1000), \mathrm{R}$ is in $\mathrm{m}$ \\
\hline \multicolumn{3}{|c|}{ Lognormal shadowing standard deviation $(\mathrm{dB})$} & 8 for $\mathrm{MCBS}^{2}, 10$ for $\mathrm{PCBS}^{1}, 10$ for $\mathrm{FCBS}^{2}$ \\
\hline \multicolumn{3}{|c|}{ Antenna configuration } & Single-input single-output for all BSs and UEs \\
\hline \multicolumn{3}{|c|}{ Antenna pattern (horizontal) } & $\begin{array}{l}\text { Directional }\left(120^{\circ}\right) \text { for } \mathrm{MC}^{1} \text { and } \\
\text { omnidirectional for } \mathrm{PC}^{1} \text { and } \mathrm{FC}^{1}\end{array}$ \\
\hline \multicolumn{3}{|c|}{$\begin{array}{l}\text { BS antenna gain plus connector loss and UE antenna } \\
\text { gain }^{2}(\mathrm{dBi})\end{array}$} & $\begin{array}{l}14 \text { for } \mathrm{MCBS}^{2}, 5 \text { for } \mathrm{PCBS}^{1}, 5 \text { for } \mathrm{FCBS}^{1}, 0 \\
\text { for } \mathrm{UE}\end{array}$ \\
\hline \multicolumn{3}{|c|}{ UE noise figure $^{2}$ and UE speed ${ }^{1}$} & $9 \mathrm{~dB}, 3 \mathrm{~km} / \mathrm{hr}$ \\
\hline \multicolumn{4}{|c|}{ Total number of MUs, UEs per FC, and multi-storage buildings 30,1 , and 1} \\
\hline \multicolumn{3}{|c|}{ PC coverage and MUs offloaded to all PCs ${ }^{1}$} & $40 \mathrm{~m}$ (radius), $2 / 15$ \\
\hline \multicolumn{3}{|c|}{$\begin{array}{l}\text { Percentage of indoor MUs }{ }^{1} \text {, external wall penetration } \operatorname{loss}^{1}\left(L_{\mathrm{ow}}\right) \text {, and } \\
{ }^{3} \text { the value of } \beta\end{array}$} & $\operatorname{sss}^{1}\left(L_{\mathrm{ow}}\right)$, and \\
\hline \multicolumn{3}{|c|}{ FC model $^{2}$ and channel state information } & 3GPP dual strip and Ideal \\
\hline \multicolumn{3}{|c|}{ Scheduler and traffic model } & Proportional Fair and full buffer \\
\hline $\mathrm{TTI}^{1}, T, \alpha_{\mathrm{on} \rightarrow \mathrm{o}}$ & scheduler constant & ${ }_{c}^{c}$, and $\alpha_{\text {off } \rightarrow \text { on }}$ & $1 \mathrm{~ms}, 8 \mathrm{~ms}, 0,100 \mathrm{~ms}$, and 0 respectively \\
\hline Simulation run & me for any FBS pa & rn $\boldsymbol{T}$ and total & ulation run time $8 \mathrm{~ms}$ and $\left(\boldsymbol{T} \times\left|\alpha_{\mathrm{fbs}}\right|\right)$ \\
\hline
\end{tabular}

taken ${ }^{1}$ from [26] , 2 from [25], and ${ }^{3}$ from [27-29].

FPP, the aggregate capacity of all MUs does not change considerably as shown in Fig. 9. Hence, the system level capacity shows also a linear response and increases with an increase in AAF of the FCBS.

Note that in Fig. 9, though the aggregate capacity of all offloaded MUs and all outdoor MUs seem to be the same, the throughput per UE of each category differs significantly from one another. Specifically, more capacity per offloaded MU than that of per outdoor MU is achieved because of less number and 
better channel condition of offloaded MUs than that of outdoor MUs. Since an indoor MU experiences a high external wall attenuation loss of a building, they are relatively less scheduled and can gain lower throughput than that of other MU categories.

The number of FBSs per FPP has a negligible impact on the aggregate capacity of all MUs (Fig. 9) and a significant impact on the capacity of FCBS (Fig. 8). Moreover, the switching delay from the transmit power on/off operation of FCBS and the UE traffic request processing delay from exchanging control signaling between the MCBS and the FCBS influence greatly the capacity of FCBS, particularly in indoor regions such as hotspots, indoor stadiums, and airports where the movement of UEs is frequent enough to cause to originate a large number of UE traffic requests for both connection and disconnection with the FCBS. All these effects are captured in Fig. 8 by varying AAF from 0 (always off) to 1 (always on) over an FPP to show how the FCBS capacity varies with a change in environment.

In Fig. 10, the transmit power saving response of the FCBS is shown. As expected, the transmit power saving decreases with an increase in AAF. This calls for a trade-off between the high capacity and the power saving demands of a FCBS. We address this issue by deriving an OAF in Eq. (27). As shown in Fig. 10, the OAF is about 0.5 , which complies with the derived OAF in Eq. (27). Hence, an optimization of both the capacity and energy efficiency of FCBSs can be achieved when each FCBS has on an average an equal or near equal on-state and off-state durations subject to $\kappa=1 / 2$. In general, for $1 / 2<\kappa<1$, the slope

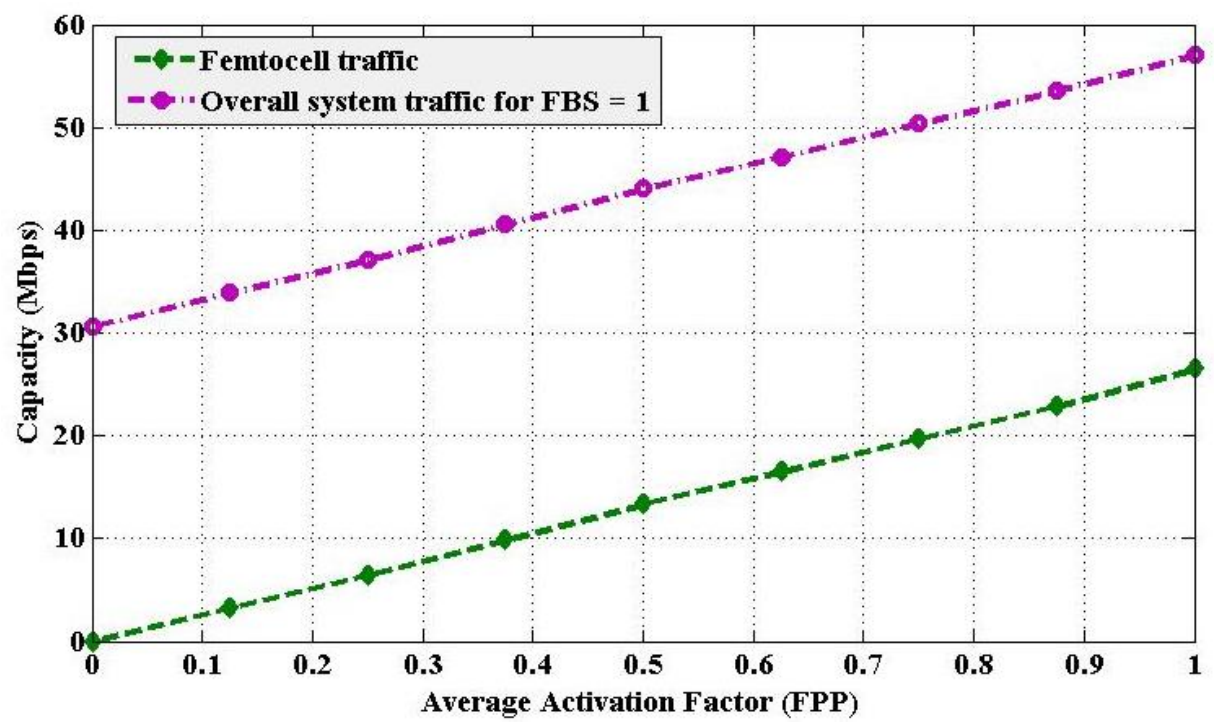

Fig. 8. Capacity with a variation in AAF.

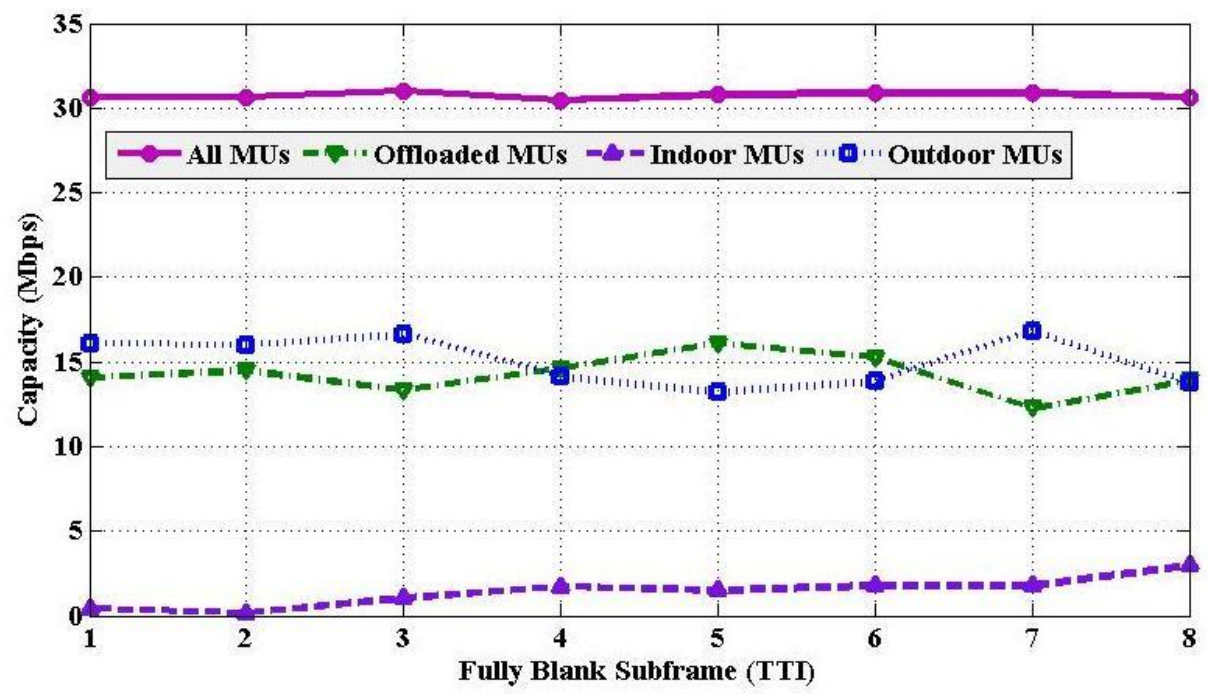


Fig. 9. Capacity of different categories of MUs with a variation in FBS.

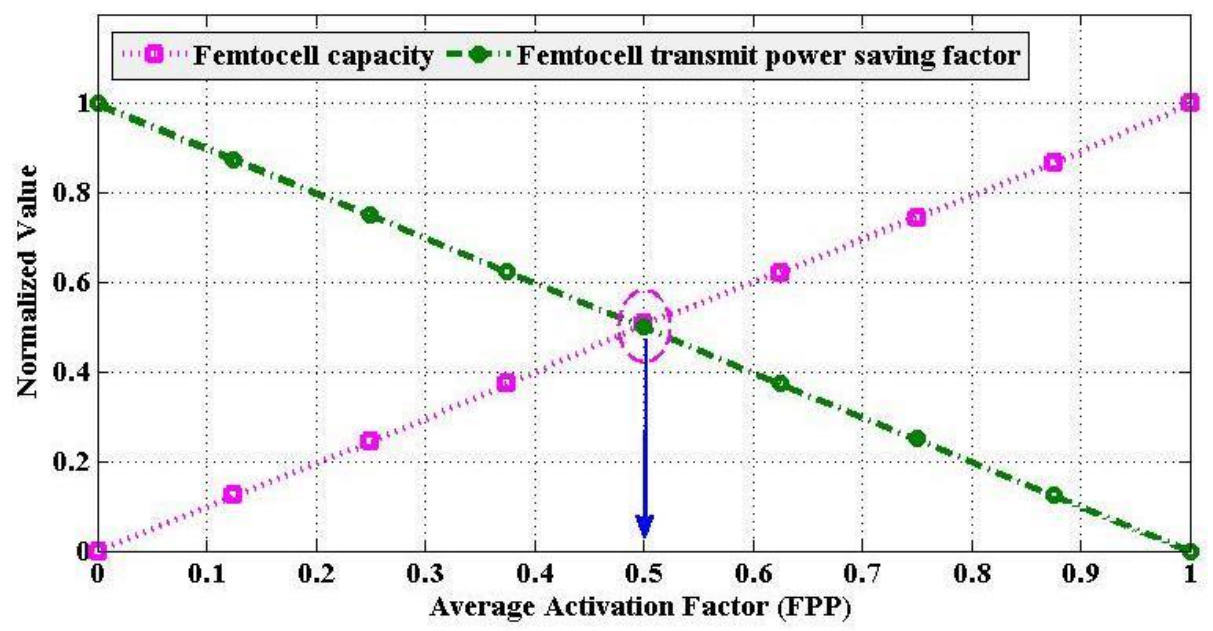

Fig. 10. Femtocell capacity, transmit power saving factor, and OAF with a variation in AAF.

of capacity of the FCBS (Fig. 10) decreases, which results in an obvious increase in the value of OAF from 0.5 in order to compensate the capacity of the FCBS corresponding to the amount of decrease in slope from that of $\sigma_{\mathrm{FC} \text {,max }}$.

Further, a FCBS can be considered serving neighboring on-state FCBSs during its normal off-state, which corresponds to its OAF $\alpha^{*}$, using JT CoMP to improve the network capacity only. In such case, a FCBS needs to operate in a CoMP set for duration greater than that corresponds to the OAF. However, if the energy efficiency is concerned only, a FCBS can operate for duration less than that corresponds to the OAF. In such case, no JT CoMP set is needed to form, and a FCBS can operate as a usual single entity. If both network capacity and energy efficiency are concerned, as mentioned already, a FCBS needs to operate for duration corresponds to the OAF.

\section{Conclusion}

In this paper, we propose a centralized 3D radio resources, namely time, frequency and power, allocation and scheduling strategy for a multi-tier control-/user-plane separation architecture (CUSA). Centralized scheduling is performed by considering schedulers of all BSs located at a central station acting as a hub for scheduling resources to all BSs. We propose a fully blank subframe (FBS) based eICIC to split completely C-/U-plane such that C-plane of all UEs can be served by the MCBS only, and U-plane of a UE by its respective BS. The cross-tier interference is avoided in time-domain, and the co-tier interference is avoided in frequency-domain by allocating frequency resources orthogonally in each tier.

The transmit power of any FCBSs is controlled by proposing a power control mechanism, modeled its on-state and off-state as conventional on/off traffic source model for single user per FCBS. However, for multiple user per FCBS, the transmit power of a FCBS is modeled as conventional Birth-Death process. We also derive an optimal value of average activation factor (OAF) over a FPP to trade-off capacity and power saving factor of any FCBSs to address both spectral and energy efficiency demands of $5 \mathrm{G}$ cellular. The performance evaluation is carried out with a multi-tier network that consists of a MCBS and a number outdoor PCBSs and indoor FCBSs deployed in a multi-storage building. The whole system bandwidth is reused in FCBSs within the building.

With a system level simulation for a single FCBS, we show that both the capacity of the FCBS and the overall system capacity increase, whereas the transmit power saving of the FCBS decreases linearly with an increase in AAF. Also, an OAF of 0.5 for $\kappa=1 / 2$, which is compliant with the derived OAF value and greater than 0.5 for $1 / 2<\kappa<1$ is found. The number of FBSs per FPP has a negligible impact on the capacity of the MCBS, whereas it has a significant impact on the achievable capacity of the FCBS. Moreover, a FCBS can also serve neighboring on-state FCBSs during its normal off-state using JT CoMP to improve the network capacity by operating at an AAF greater than the $\mathrm{OAF}$, whereas operating at an AAF 
lower than the OAF as a usual single entity if the energy efficiency needs to improve. This contribution will give operators insights on scheduling time, frequency, and power centrally particularly for SCs in CUSA in order to address the demands of the high indoor capacity and energy efficiency of future $5 \mathrm{G}$ mobile networks.

\section{Acknowledgements}

The initial results of this paper were presented partly in the conference article [30]. The research grant funds have been provided by "The $100^{\text {th }}$ Anniversary Chulalongkorn University Fund for Doctoral Scholarship" upon submission of application of research expenses for "The $90^{\text {th }}$ Anniversary Chulalongkorn University Fund (Ratchadaphiseksomphot Endowment Fund)" and "The Electrical Engineering Chulalongkorn University PhD (EECU-PhD) Honors Program Scholarship" at Chulalongkorn University, Bangkok, Thailand. The research is also graciously supported by the STAR Project, Department of Electrical Engineering, Chulalongkorn University, Thailand.

\section{References}

[1] H. T. Cheng and W. Zhuang, "Joint power-frequency-time resource allocation in clustered wireless mesh networks," IEEE Netw., vol. 22, no. 1, pp. 45-51, Jan./Feb. 2008.

[2] L. Zhao, L. Cong, F. Liu, K. Yang, and H. Zhang, "Joint time-frequency-power resource allocation for low-medium-altitude platforms-based WiMAX networks," IET Commun., vol. 5, no. 7, pp. 967-974, 2011.

[3] D. T. Ngo, S. Khakurel, and T. Le-Ngoc, "Joint subchannel assignment and power allocation for OFDMA femtocell networks," IEEE Trans. Wireless Commun., vol. 13, no. 1, pp. 342-355, Jan. 2014.

[4] M. Qian, Y. Wang, Y. Zhou, L. Tian, and J. Shi, "A super base station based centralized network architecture for 5G mobile communication systems," Digital Commun. \& Netw., vol. 1, no. 2, pp. 152159, 2015.

[5] China Mobile Research Institute, "C-RAN the road towards green RAN," White Paper, ver. 2.5, Oct. 2011.

[6] Y. Lin, L. Shao, Z. Zho, Q. Wang, and R. K. Sabhiki, "Wireless network cloud: Architecture and system requirements," IBM J. Research \& Development, vol. 54, no. 1, pp. 4:1-4:12, Jan.-Feb. 2010.

[7] Alcatel-Lucent, "LightRadio," White Paper: Technical Overview, 2011.

[8] X. Duan, A. M. Akhtar, and X. Wang, "Software-defined networking-based resource management: data offloading with load balancing in 5G hetnet", EURASIP J. Wireless Commun. \& Netw., vol. 181, pp. 1-13, 2015.

[9] F. Zhang, S. Sun, N. Chen, and H. Huang, "Radio resource management based on NFV and SDN in 4G and 5G hetnet," S. Sun and J. Li (Eds.) Self Organizing Networks, ICSON 2015, Lecture Notes of the Institute for Computer Sciences, Social Informatics and Telecommunications Engineering, Vol. 149, pp. 165172, 2015.

[10] A. Prasad, A. Maeder, and C. Ng, "Energy efficient small cell activation mechanism for heterogeneous networks," in Proc. Globecom Workshop-Heterogeneous and Small Cell Networks, 2013, pp. 754-759.

[11] C. Liu, B. Natarajan, and H. Xia, "Small cell base station sleep strategies for energy efficiency," IEEE Trans. Veh. Technol., vol. 65, no. 3, pp. 1652-1661, Mar. 2016.

[12] G. Lee and H. Kim, "Green small cell operation using belief propagation in wireless networks," in Proc. Globecom Workshop-Heterogeneous and Small Cell Networks, 2014, pp. 1266-1271.

[13] R. K. Saha, P. Saengudomlert, and C. Aswakul, "Evolution toward 5G mobile networks - A survey on enabling technologies," Engineering Journal, vol. 20, no. 1, pp. 87-119, 2016.

[14] R. K. Saha and C. Aswakul, "Incentive and architecture of multi-band enabled small cell and UE for up-/down-link and control-/user-plane splitting for 5G mobile networks," Frequen₹J. RF-Engineering \& Telecommun., vol. 75, no. 1-2, pp. 95-118, Nov. 2016.

[15] H. A. U. Mustafa, M. A. Imran, M. Z. Shakir, A. Imran, and R. Tafazolli, "Separation framework: An enabler for cooperative and D2D communication for future 5G networks," IEEE Commun. Surveys \& Tuts., vol. 18, no. 1, pp. 419-445, 2016. 
[16] A. Mohamed, O. Onireti, M. A. Imran, A. Imran, and R. Tafazolli, "Control-data separation architecture for cellular radio access networks: a survey and outlook," IEEE Commun. Surveys \& vol. 18, no. 1, pp. 446-465, 2016.

[17] J. D. Chimeh, M. Hakkak, and S. A. Alavian, "Internet traffic and capacity evaluation in UMTS downlink," in Proc. Future Generation Communication and Networking (FGCN), 2007, pp. 547-552.

[18] ETSI, "Universal mobile telecommunications system (UMTS); selection procedures for the choice of radio transmission technologies of the UMTS," European Telecommunications Standards Institute (ETSI), TR 101 112,UMTS 30.03, ver. 3.2.0, 1998-2004.

[19] L. Kleinrock, Queueing Systems, Vol I: Theory. Wiley-Interscience, 1975.

[20] J. Perez-Romero, O. Sallent, R. Agusti, and M. A. Diaz-Guerra, Radio Resource Management Strategies in UMTS. Wiley, 2005.

[21] 3GPP, "Technical specification group radio access network; evolved universal terrestrial radio access (E-UTRA); FDD home eNodeB (HeNB) radio frequency (RF) requirements analysis (release 9)," $3^{\text {rd }}$ Generation Partnership Project (3GPP), TR 36.921, Ver. 2.0.0, Mar. 2010.

[22] J. Ellenbeck, J. Schmidt, U. Korger, and C. Hartmann, "A concept for efficient system-level simulations of OFDMA systems with proportional fair fast scheduling," in Proc. IEEE GLOBECOM Workshops, 2009, pp. 1-6.

[23] Y. Wang, K. I. Pedersen, T. B. Sørensen, and P. E. Mogensen, "Carrier load balancing and packet scheduling for multi-carrier systems," IEEE Trans. Wireless Commun., vol. 9, no. 5, pp. 1780-1789, 2010.

[24] H. J. Bang, T. Ekman, and D. Gesbert, "Channel predictive proportional fair scheduling," IEEE Trans. Wireless Commun., vol. 7, no. 2, pp. 482-487, 2008.

[25] 3GPP, "Simulation assumptions and parameters for FDD HeNB RF requirements," 3rd Generation Partnership Project (3GPP) TSG RAN WG4 (Radio) Meeting \#51, R4-092042, May 2009.

[26] 3GPP, "Evolved universal terrestrial radio access (E-UTRA); radio frequency (RF) system scenarios," $3^{\text {rd }}$ Generation Partnership Project (3GPP), TR 36.942, ver. 1.2.0, Jul. 2007.

[27] R. K. Saha and P. Saengudomlert, "Novel resource scheduling for spectral efficiency in LTEAdvanced systems with macrocells and femtocells," In Proc. International Conference on Electrical Engineering/Electronics, Computer, Telecommunications and Information Technology(ECTI-CON), pp. 340-343, 2011.

[28] R. K. Saha and C. Aswakul, "A tractable analytical model for interference characterization and minimum distance enforcement to reuse resources in three-dimensional in-building dense small cell networks," Int. J. Commun. Sys.,(early view) pp. e3240, 2017, doi: 10.1002/dac.3240

[29] R. K. Saha, "Modified proportional fair scheduling for resource reuse and interference coordination in two-tier LTE-Advanced systems," Int. J. Digital Info.\& Wireless Commun., vol. 3, no. 2, pp. 9-28, Jul. 2013.

[30] R. K. Saha and C. Aswakul, "Centralized 3D radio resource allocation and scheduling for multi-tier control-/user-plane split architecture for 5G mobile network," in Proc. 2017 International Conference on Electronics, Information, and Communication (ICEIC), (to appear in IEEE Xplore), Phuket, Thailand, 11-14 Jan. 2017, pp. 530-533. 